\title{
BRCA1 NP_009225.1:p.Q1756Pfs
}

National Cancer Institute

\section{Source}

National Cancer Institute. BRCA1 NP 009225.1:p.Q1756Pfs. NCI Thesaurus. Code C131476.

A change in the amino acid sequence of breast cancer type 1 susceptibility protein where an insertion mutation results in the replacement of the glutamine at position 1756 with a proline immediately followed by a stop codon. 